Article

\title{
Evidence Falsifying the Double Helix Model
}

\author{
You Cheng $\mathrm{Xu}$ \\ UT Southwestern Medical Center at Dallas, Dallas, TX 75390, USA; ycxu2011@gmail.com
}

Received: 15 October 2019; Accepted: 14 November 2019; Published: 25 November 2019

\begin{abstract}
Through more than 40 years of reading, thinking, searching, and experimentation, we have found that the double helix model carries some defects or incorrect information. Evidence gleaned from the literature clearly indicates that the two strands of DNA are coiled ambidextrously, rather than plectonemically. It is likely that the linking number of native chromosomal Escherichia coli (E. coli) DNA is less than 960. Presently, a clear voice is necessary to break the ice formed from decades of misleading media, questionable textbooks, and expediency. For the sake of science, we are responsible and willing to share our hard-earned knowledge, experience, and knack with the public. A promising research plan is provided for the additional falsification of the right-handed double helix model. It would be a precision hit at the Achilles' heel of the double helix model. An appropriate conceptual shift will hopefully lead to new knowledge on the secondary structure of DNA and improve understanding of its biological functions.
\end{abstract}

Keywords: linking number; topoisomerase; left-handed DNA; positive supercoiling; super helical index; ambidextrous double helix

\section{Introduction}

\subsection{The Proposal of the Double Helix Model is a Great Progress in Molecular Biology}

Accompanying the appearance of life on this planet, DNA molecules emerged about 4 billion years ago.

Starting in 1869, Miescher found a relatively pure sample from pus. His further analysis made it clear that it contained lots of phosphorus and it was different from carbohydrates or protein. He called it nuclein, but its detailed chemistry and biological significance was not understood for many decades.

After many years of investigation by scientists all over the world, our knowledge of DNA has gradually increased through a very long and twisted road. Presently, we know much more about DNA than before; however, many unanswered questions remain. Just as Crick et al. stated: "DNA is such an important molecule that it is almost impossible to learn too much about it." [1].

Depending on the base pairing, DNA fiber X-ray analysis, model building, and the information available at that time, Watson and Crick proposed a hypothesis in 1953 [2]. The self-replicating ability of the double helical structure of the DNA molecules aroused great interest and excitement. Many scientists rushed into this field and generated many important discoveries. The finding of the double helix is no doubt a milestone in DNA investigation and it is a great progress in molecular biology. Anyone daring to argue the validity of the double helix may be deemed as committing blasphemy against modern science.

\subsection{The Evidence Supporting the Watson-Crick Model is Not Enough}

In the Watson-Crick model, the double helix is composed of two intertwined, anti-parallel complementary strands held together by hydrogen bonds between corresponding base pairs. These properties were proven by many experiments afterwards. However, the proof of the right-handed 
winding is scant. Rich pointed out that a few diffraction spots obtained from DNA fiber x-ray analysis is not enough to prove the structure of DNA [3].

The winding direction inside the double helix is very difficult to test or examine. No reliable method is available to detect it. The resolution of the most advanced electron microscope today is still not good enough to perceive the detailed molecular structure of a plasmid or chromosome clearly.

The untangling problem was probably first asked by Max Delbrück in 1953: "I am willing to bet that the plectonemic coiling of the chains in your structure is radically wrong, because (1) the difficulties of untangling the chains do seem, after all, insuperable to me. (2) The X-ray data suggest only coiling but not specifically your kind of coiling." [4].

Actually, DNA untangling had worried Watson and Crick for decades. In 1983, when Watson learned that the double helix was proved using X-ray crystallography, he called Rich and said that he had his first good night's sleep in 20 years [3]! In Crick's words in What a Mad Pursuit: "He (Watson) suffered from periotic fears that the structure might be wrong and that he had made an ass of himself." [5] (p. 66).

After the finding of topoisomerase, Crick became confident on the correctness of the double helix as he wrote in What a Mad Pursuit: "The double-helical structure of DNA was thus finally confirmed only in the early 1980s. It took over twenty-five years for our model of DNA to go from being rather plausible, to being very plausible (as a result of the detailed work on DNA fibers), and from there to being virtually certainly correct." [5].

\subsection{The Untangling Problem of the Double Helix Became More Severe After a Series of Findings}

The experiment of Meselson and Stahl indicated that the two parental strands have to be completely separated after each replication round of replication [6]. Their experimental result was accepted as a general rule where the DNA replication in E. coli and many other bacteria are all carried out in a semi-conservative, bi-directional mechanism.

The finding of circular chromosomal DNA and many plasmids in E. coli definitely frustrated many molecular biologists, since if the two complementary circular rings are always tightly tangled with each other, for topological reasons, their separation would be very difficult [7-9].

According to the double helix model, the linking number of E. coli chromosomal DNA should be a very big integer, i.e., $4.6 \times 10^{5}$ [10]. Replication needs this linking number to drop to exactly zero. The replisome, a complex molecular machine that carries out DNA replication, presumably separates the two intertwined parental strands at the rate of $100 \mathrm{rps}$ or $6000 \mathrm{rpm}$ [11].

Every experienced biochemist knows that in vitro, the long DNA molecules could be easily broken by a mild vortex or gentle stir. It is questionable whether the quick unwinding reaction really occurred in the viscous cytosol where friction is very high. Actually, nobody knows if this quick unwinding really happened during replication.

In order to prevent or resolve the unwinding problem during replication, several DNA models, including the cis ladder conformation [12] "side by side" (SBS) [13] and Corey-Pauling-Kortum (CPK) structure models [14] were proposed. All these DNA models were proposed according to imagination based on structural chemistry and base pairings but with no new evidence. It is interesting to note that a vague electron microscope (EM) picture seems similar to the image of the hypothetical CPK model.

In response to various alternative DNA models raised in the late 1970s, Crick et al. wrote a review in 1979 [1]. They pointed out a clever way to judge the conflicting scientific arguments: "In science ten weak arguments do not add up to one strong one." According to the evidence available in 1979, they were pretty sure that the right-handed double helix model is correct and the problem in untangling the double helix can rely on the strand-passing ability of topoisomerases. However, only several weak arguments were provided in their review supporting the plectonemic double helix model. Hence, they admitted that the double helix model is suggestive but not conclusive. That is why they wisely proposed a possible reservation, i.e., if dramatic experimental evidence is provided that the paired circular strands of a plasmid can be physically separated, that may be strong enough to admit the 
presence of an alternative double helix structure. They suggested that this should and could be done by raising the temperature until the structure denatured. Perhaps someone had tried that kind of experiment and failed. The fact that this evidence is missing might be the evidence supporting the double helix model.

\subsection{How the Double Helix is Untangled Remains an Unanswered Question}

Presently, under physiological conditions, the structure of native DNA is generally assumed to be in the B-DNA form, and it is written as a doctrine in almost all textbooks. Most people did not think there was any problem in the double helix and most scientists considered that the untangling problem has been solved by topoisomerases. However, this qualitative guess was defeated by quantitative analysis many years later. In 1996, Ullsperger and Cozzarelli found that in E. coli, gyrase is mainly responsible for the unwinding of the double helix; its reaction rate is only six times per minute [15]. In E. coli, each replisome at the replication fork contains one gyrase and one helicase operating for the opening of the complementary chromosomal DNA. Since the linking number changed in one gyrase catalytic reaction is 2 and each replication round takes $40 \mathrm{~min}$, the total linking number change by gyrase at both forks is only $2 \times 40 \times 6 \times 2=960$. This is too slow to catch up with the unwinding speed of the DNA replication $(6000 \mathrm{rpm})$ required by the double helix model [15]. This measured slow reaction rate of gyrase is reliable and reasonable because the catalytic strand passing reaction is rather complicated, and its reaction rate cannot be as fast as most of those simple enzyme catalytic reactions [16]. Thus, how the two strands of DNA are untangled remains unanswered. It is likely that the linking number of native E. coli chromosomal DNA is less than 960.

In general, the untangling problem is supposed to be solved by the helicase and gyrase in each replisome, a complicated DNA replication factory located at the replication fork. With the energy derived from ATP hydrolysis, helicase is able to quickly open the hydrogen bond. However, no evidence indicates that helicase changes the linking number. If the DNA is right-handed, helicase will push the two parental strands to twist tighter in front of the replication fork. This would impede the advancement of the replication fork. Due to the slow reaction rate of gyrase, it is unlikely that the two parental strands can be truly separated.

The mechanism of DNA replication is one of the most complicated processes in molecular biology. After many years of investigation by many scientists, all the components involved in E. coli replication and its main procedures were clarified with solid evidence [17].

Bacteriology tells us that the doubling time of E. coli, a model organism in life science research, is $20 \mathrm{~min}$ under the best physiological conditions, and one round of its chromosomal DNA replication needs $40 \mathrm{~min}$. From the view point of topology and the knowledge of biochemistry, how the linking number drops from $4 \times 10^{5}$ to exactly zero is an unavoidable and difficult topological and biochemical question.

The fact is that E. coli and other organisms have lived on Earth for millions of years and their replication has never encountered any difficulty. Hence, untangling of the DNA double helix becomes a great challenge to our wisdom.

Since the supposed quick unwinding problem results from the double helix model, and this quick unwinding has never been proved with solid evidence, it reasonably leads to doubting the plectonemic winding of the double helix model.

\section{Results}

\subsection{The Beginning of the Search}

The mystery of DNA replication greatly evokes our curiosity. Starting more than 40 years ago, we focused our attention on the untangling of the double helix. During the early stage of our investigation, through extensive literature searches and careful reading, an unusual phenomenon attracted our attention. On an EM picture, the two strands of denatured pseudoalteromonas virus 2 
(PM2) DNA, a circular bacteriophage DNA with 10,079 bp, are loosely tangled with each other [18]. Even though the authors of the paper did not give any explanation for the cause of those images, they greatly sparked our imagination.

Our inspiration led us to ponder that the physical separation of the two circular strands could imply that the two strands of DNA were not tightly tangled with each other as is written in textbooks.

Therefore, we started an independent experimental investigation and the preliminary tests proved that our idea that the DNA is not plectonemic is supported. The EM evidence helped us to propose a hypothesis that left-handed and right-handed DNA can coexistent in the same native plasmid molecule [19]. Additional investigations serendipitously found the linking number of relaxed pBR322 DNA is very low, confirming our working hypothesis [20].

The impact of our two publications on this subject remains negligible. This is probably because in the worldwide chorus of the double helix, it is barely noticeable and considered heretical. Inspired by great curiosity, pushed by an intense hunch, and supported by our experimental findings, we searched for more solid evidence. It is believed that, except in mathematics, most scientific problems cannot be solved by discussion or debate; evidence is more effective and important than eloquence. Besides, scientific knowledge should be verified by time rather than by God, expert, authority, or public opinion.

\subsection{A Different Strategy was Adopted in Searching the Answer of the DNA Untangling Problem}

Karl Popper reminds us that: "A theory which is not refutable by any conceivable event is non-scientific. Irrefutability is not a virtue of a theory (as people often think) but a vice".

We feel it is proper to adopt a different strategy from that which is commonly used to solve the untangling problem, i.e., to find the experimental evidence that can falsify the double helix, in other words, to prove the two strands of DNA are not winding plectonemically.

A disproof is stronger than thousands of proofs, no matter whether it originates from a knowledgeable scholar or from an amateur. Though even millions of white swans have been observed, this is insufficient to conclude that all swans are white.

According to the double helix model, the linking number of any plasmid with thousands of base pairs should be a very big integer. Hence, finding a non-linked plasmid would be theoretically impossible and practically unachievable. In contrast, based on our hypothesis and practice, finding a zero-linking-number topoisomer is theoretically possible and practically feasible. This double helix conjecture was clearly proposed in 2011 and we believe it can be proven experimentally [21].

\subsection{There is Much Evidence Falsifying the Plectonemic Double Helix Model}

\subsubsection{A Zero-Linking-Number-Topoisomer Can be Located on the Agarose Gel}

In retrospect, although our experiments reported in the early 1980s were rather simple and brief, the main idea that the two strands in the DNA are not always tangled plectonemically is correct $[19,20]$. Further investigation found that the linking number of each relaxed topoisomer, purified from the separated bands on agarose gel electrophoresis (AGE), is constant and the neighboring bands differ by one [22]. This test result is consistent with the speculation of Crick et al. [1]. By measuring the linking number of three different topoisomers, a zero-linking-number topoisomer was located on the agarose gel. Figure 1 represents a schematic drawing [22]. 


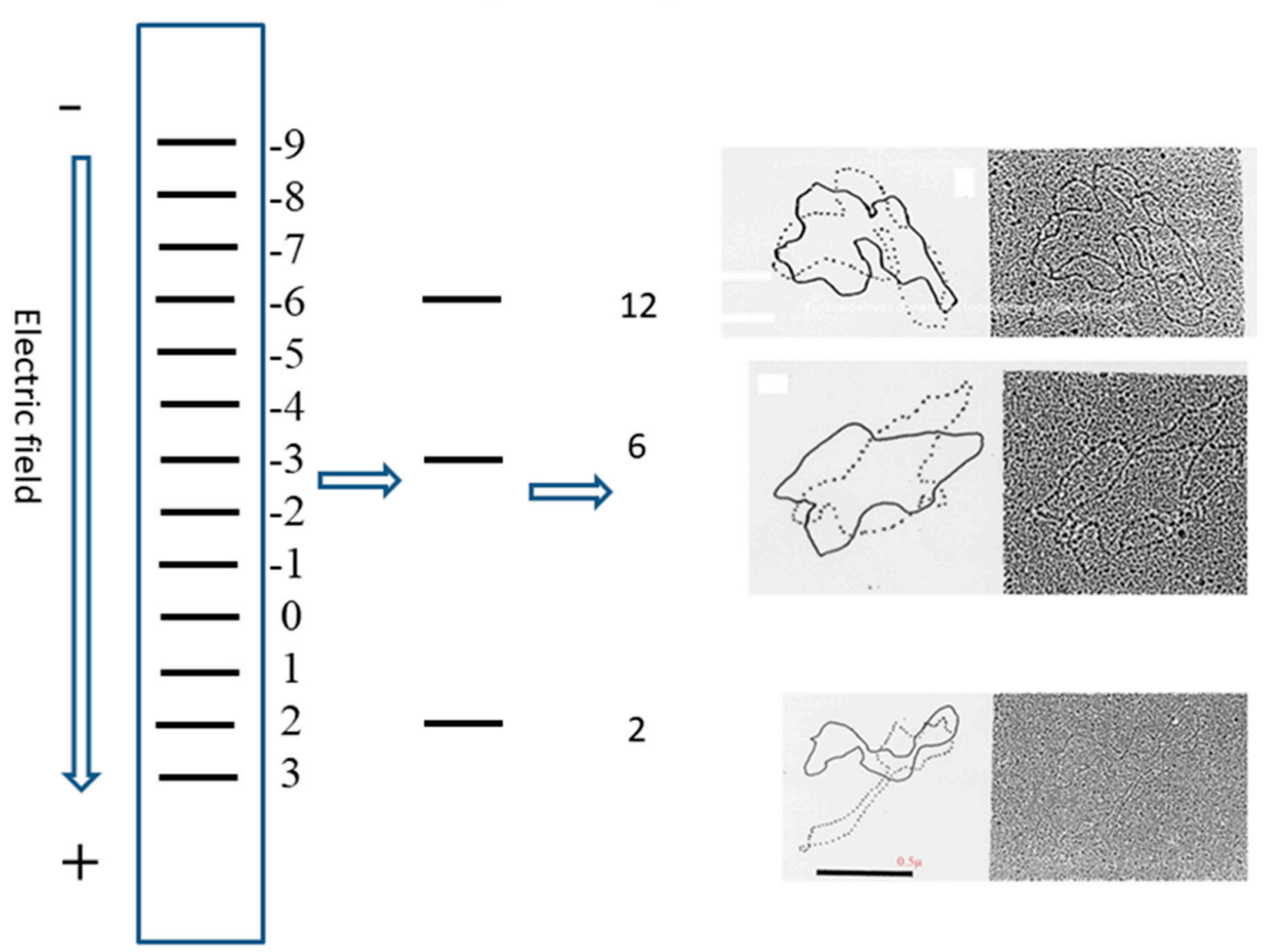

Figure 1. Schematic procedures to locate the zero-linking-number topoisomer.

\subsubsection{A Zero-Linking-Number Topoisomer Can be Made from Two Single Stranded (ssc) DNA}

Many scientists hesitated to accept the result observed on EM pictures. In order to convince them, a different test was carried out. When two complementary ssc DNAs of a plasmid, purified using alkaline sucrose gradient centrifugation, were annealed under suitable conditions, three products were produced and can be separated by AGE, as shown in Figure 2. One is nicked DNA and another one is DNA V, as reported previously by Stettler et al. [23]. A new double-stranded circular DNA was found in this test that is similar to a relaxed topoisomer of the same plasmid [22]. Since the annealing of ssc DNA is a purely biophysical process with no strand-passing reaction, this new annealing product must be non-linked DNA.

\subsubsection{The Figure Eight Test Proved the Two Strands of DNA are Winding Ambidextrously}

According to advice from J.C. Wang, a different test was carried out to find the winding information of two strands in DNA. As shown in the schematic Figure 3, the annealing product of two phage DNAs, each harboring one of the complementary strands from a $\lambda$ DNA $2 \mathrm{~kb}$ fragment, can yield a figure eight or $\theta$ structure, which can be clearly seen under EM. It is interesting to note that the annealing of the $\lambda$ $2 \mathrm{~kb}$ fragment did not push the single-stranded phage DNA to become tightly tangled [22]. If the $\lambda 2 \mathrm{~kb}$ fragment is a right-handed double helix, a topological rule suggests this would push the two phage DNAs to turn 200 times in a left-handed direction. This expectation evidently contradicts the observed fact that all the annealing products are in the form of a figure eight structure. 


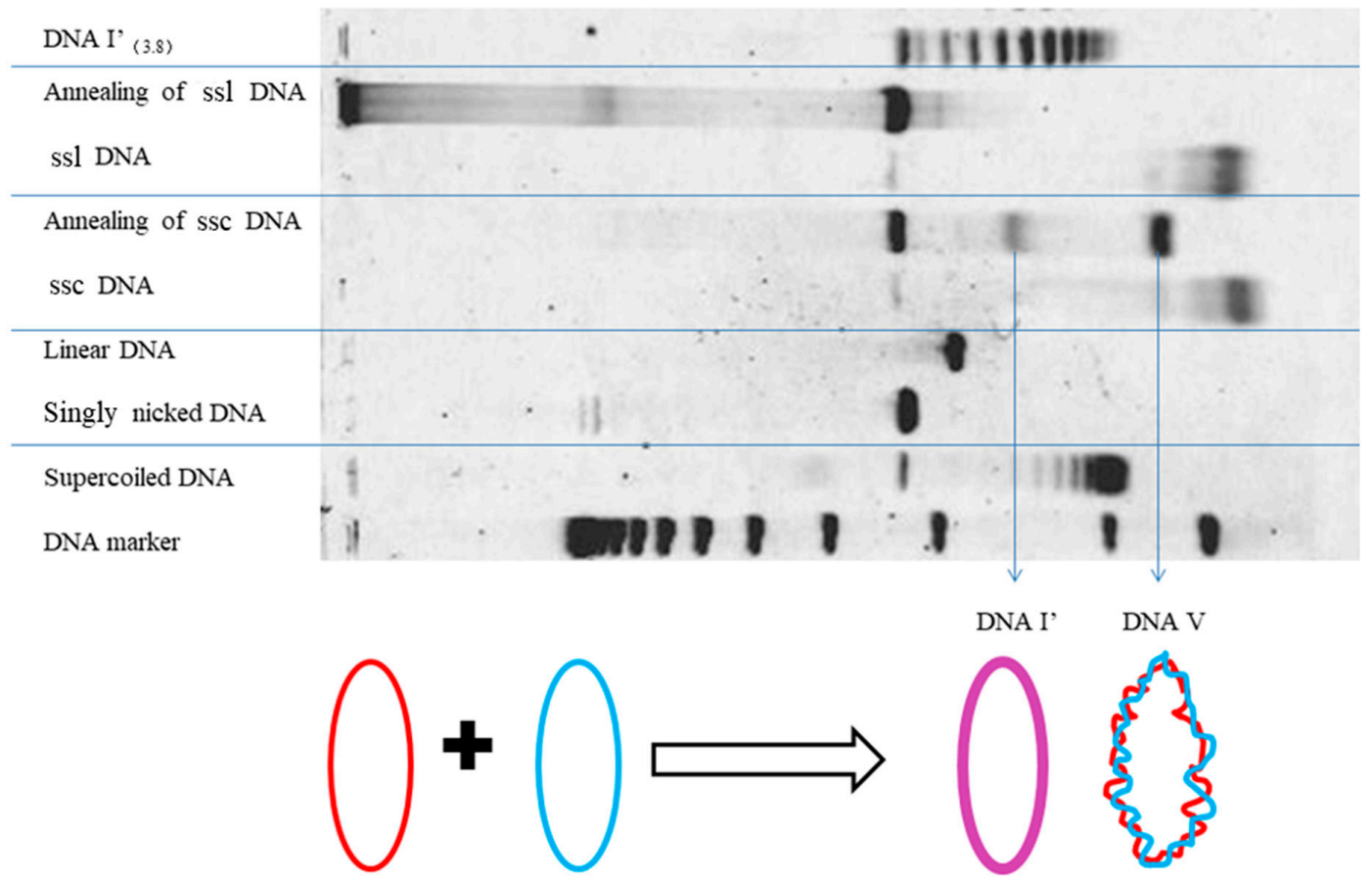

Figure 2. Schematic detailing the annealing products of two single stranded circular (ssc) DNAs with the control of single stranded linear (ssl) DNAs.
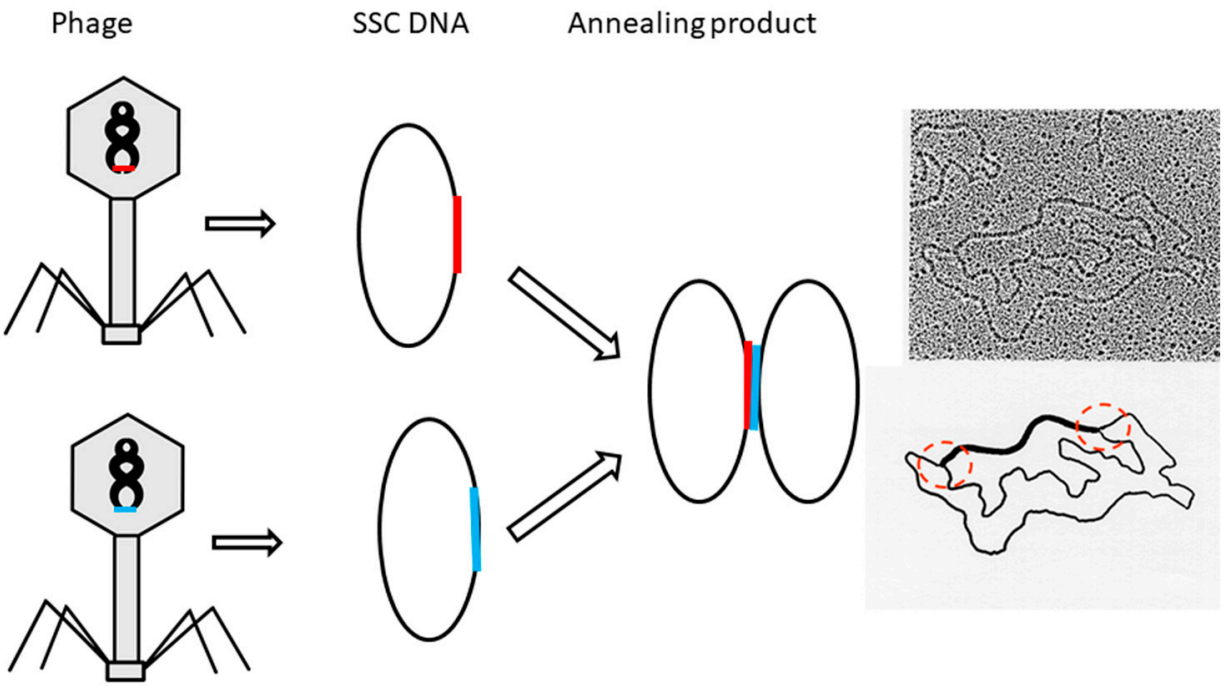

Figure 3. Schematic detailing the formation of a figure eight structure.

\subsubsection{The Two Strands of a Singly Nicked Plasmid Can Be Quickly Separated Using Alkaline}

According to the double helix model, the two strands of a plasmid are always tangled several hundred times or more. When a singly nicked plasmid is denatured by alkaline, all the hydrogen bonds were broken immediately. It would be very difficult to separate the two complementary strands since the two tangled strands need to unwind more than one hundred times. Although both ends of the broken strand are free to unwind, their movement is greatly limited by the ssc DNA, which is not always widely expanded and waiting for the broken ends of the linear DNA to pass through. Besides, the two ends of the broken strands are supposed to unwind in the opposite orientation, which would inevitably cause them to collide, as shown in schematic Figure 4. Our experimental result indicated that the two strands of singly nicked plasmid (pBR322) can be easily separated using AGE quickly after 
alkaline denaturation [22]. The plectonemic double helix model fails to give reasonable explanations for these observations, which are easily predicted by the ambidextrous double helix model.

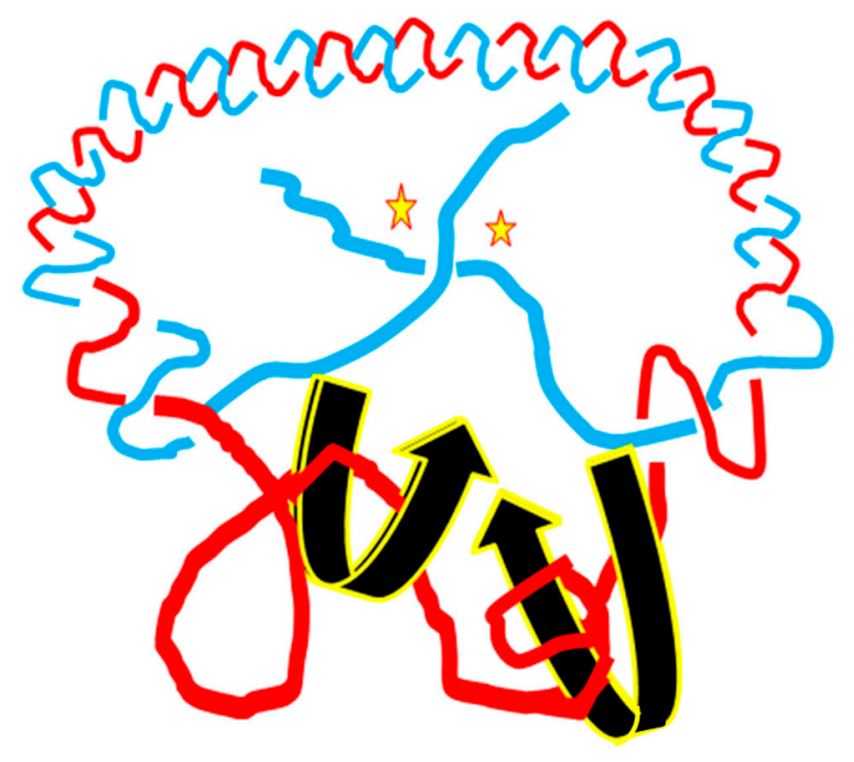

Figure 4. Schematic detailing the unwinding of a singly nicked plasmid.

2.3.5. The Topological Transformation of a Covalently Closed Circular DNA Revealed That the Two Strands of DNA Are Winding Ambidextrously

In solution, the two complementary strands are normally held by hydrogen bonds. The repelling force of negatively charged phosphor atoms on two strands is normally shielded by cation ions and is generally not noticeable. By manipulating the two antagonistic forces of DNA appropriately, an interesting phenomenon was found: the two ssc DNAs of a covalently closed circular DNA can be denatured in a completely different way and lead to the disappearance of the DNA sample on the agarose gel after AGE [24]. According to the principle of mass conservation, DNA samples cannot really disappear. They just become invisible or undetectable by standard methods. A schematic drawing is presented in Figure 5.

As detailed in our online article [24], the two strands of supercoiled pBluescript DNA can be denatured in pure water or low salt aqueous solution at elevated temperatures. As soon as the repelling force of nearby phosphor atoms surpasses the binding force of hydrogen bonds between the two DNA strands, their separation is unavoidable.

This phenomenon cannot be explained by the canonical double helix model because if the two ssc DNAs are tightly tangled with each other, as required by the Watson-Crick model, after denaturation, they would turn into compact random coils that are easily detectable on agarose gel after AGE. However, if the two ssc DNAs are not really tightly tangled with each other, their denatured product would have more freedom to move and the plasmid produces various different intermediate forms that disperse on the agarose gel along the electric field track during AGE. This could reasonably explain the disappearance of the plasmid.

Since the denaturing method we used was a relatively gentle and slow process, no nicking or breaking of the backbonds of the plasmid was found. However, there was some indication that the denatured paired ssc DNAs can be renatured, which paves the way for the experimental testing of the double helix conjuncure. 


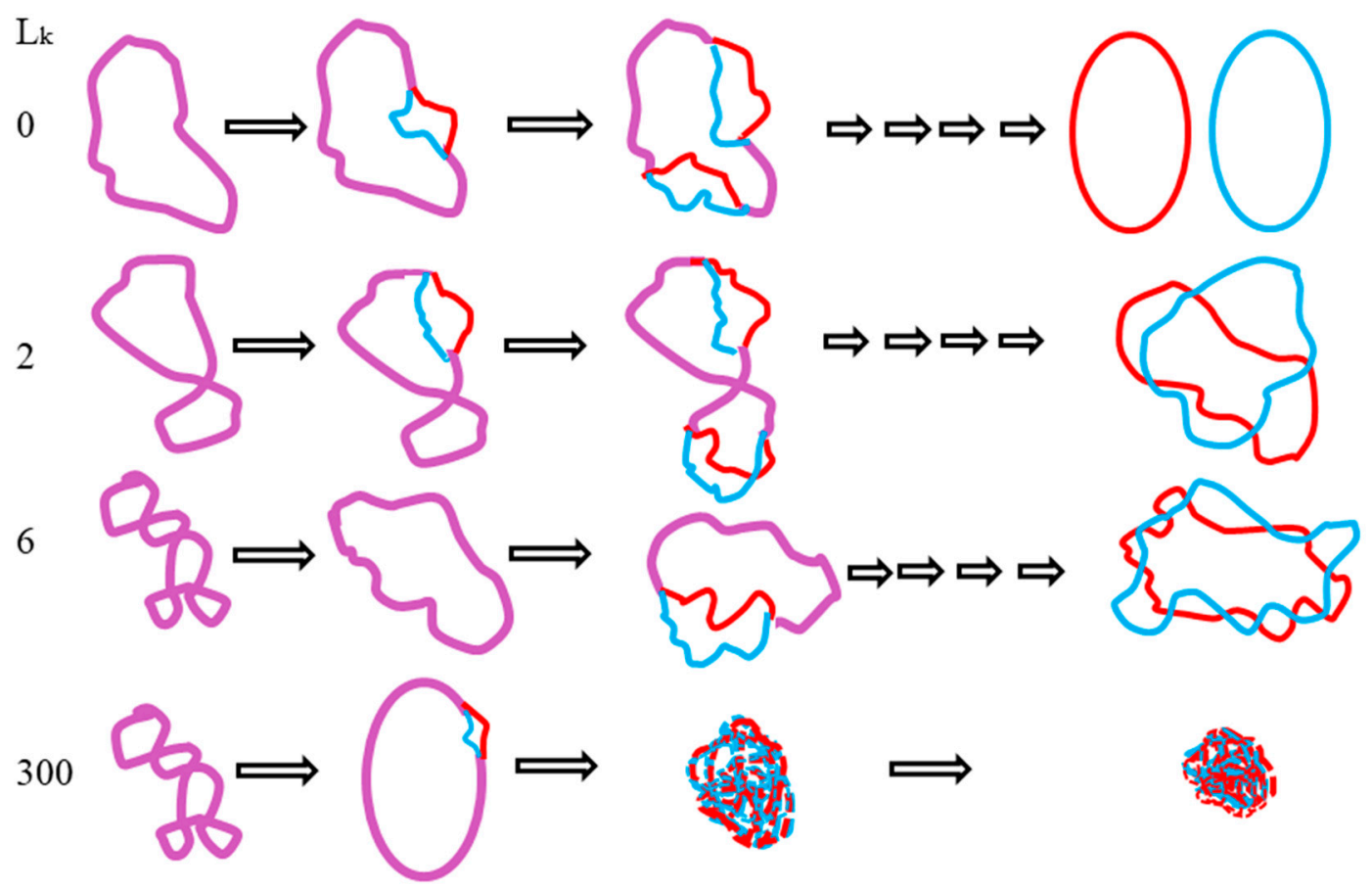

Figure 5. Schematic detailing the topological transformation of closed circular DNA.

\subsection{Many Facts Falsified the Right-Handed Double Helix Model}

It is interesting to note that many facts, found by many different scientists, had already objectively falsified the right-handed double helix model, although these authors did not intend to disprove the double helix.

There might be many more similar unnoticed cases in the literature we did not notice; here are just a few examples of the most obvious facts.

(a) In 1972, Dalius et al. [25] published an EM picture showing that the two complementary strands of $\lambda$ DNA can be gradually torn apart by gene 32, a molecular weight 35,000 Da single-strand binding protein (SSB), to form two untangled parallel lines, as shown in the schematic picture of Figure 6. Each gene 32 protein binds 10 nucleotides of ss DNA, preferentially located at AT-rich regions or single stranded terminals in a cooperative way. For some reason, the authors did not claim this phenomenon actually provides clear visual evidence that the two strands of DNA are not tightly tangled with each other.

(b) It was reported that the length of $\lambda$ DNA can be stretched to twice its normal size $[26,27]$, as shown in the schematic drawing of Figure 7.

This fact cannot be explained by the Watson-Crick model since the axial distance of neighboring phosphate atoms $(3.4 \AA)$ in each strand are almost stretched to their limit. Just before breaking, all the phosphate atoms of the $\lambda$ DNA need to be lined in a straight line. It is unlikely that the two complementary strands can remain tightly tangled and stretched to double their original length simultaneously.

(c) Freifelder and Davidson [28] found that T7 DNA, a 39,936 bp linear DNA, can be physically separated using formaldehyde. If the T7 DNA is in the canonical double helix structure, the two thin strands should tangle about 4000 times. To separate them in solution is not an easy job. 


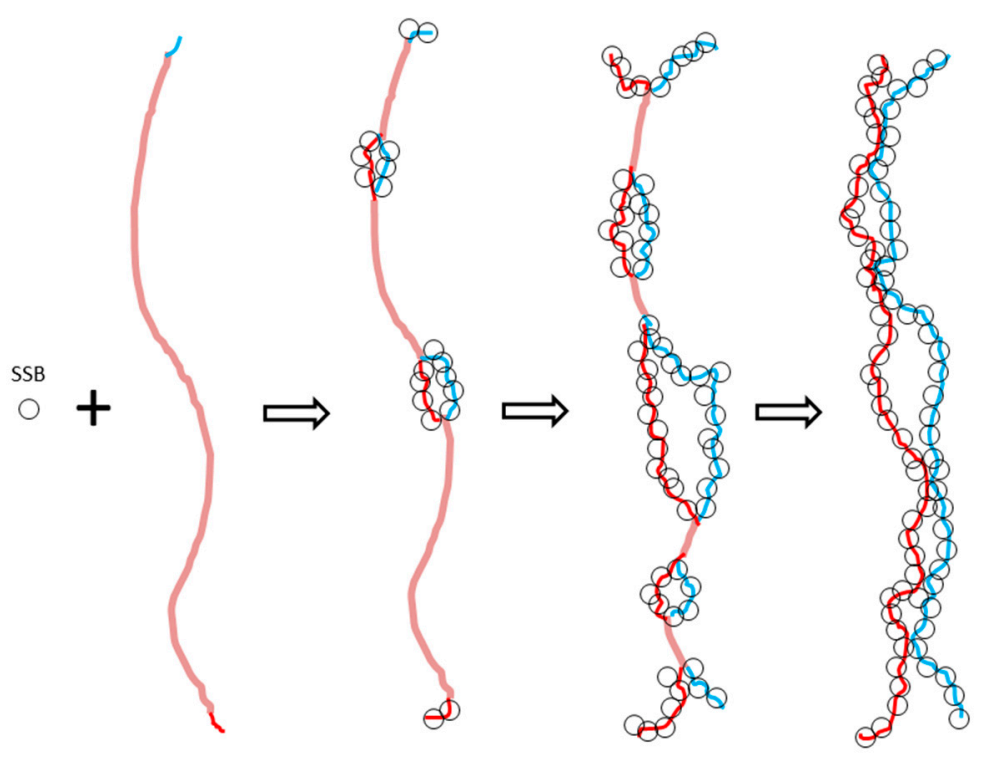

Figure 6. Schematic showing the tearing apart process of the two complementary strands of $\lambda$ DNA by a single-strand binding protein (SSB).

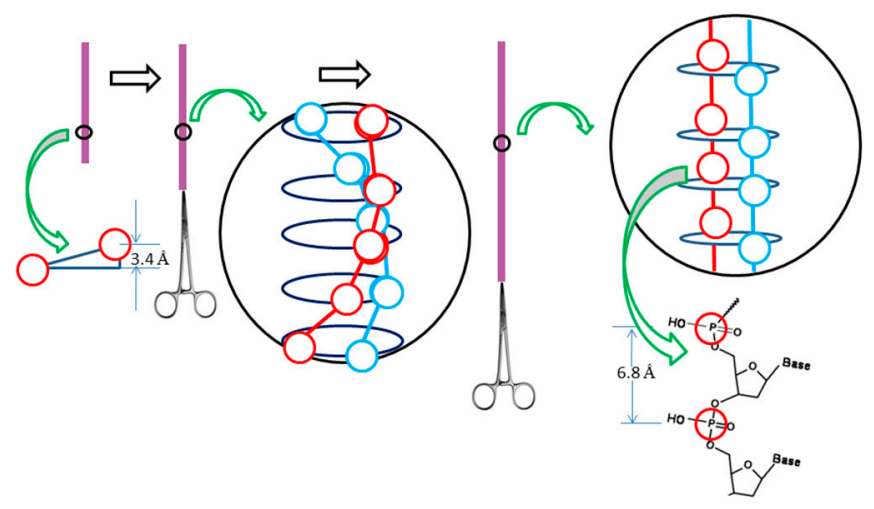

Figure 7. Schematic detailing the stretching process of $\lambda$ DNA.

The schematic drawing Figure 8 shows, as the temperature increases, the two strands of T7 DNA gradually separated from each other. At $54{ }^{\circ} \mathrm{C}$, the two strands completely separated and the sedimentation coefficient dropped abruptly.

(d) McKay and Steitz [29] demonstrated that the structure of the catabolite gene activator protein (CAP) at a resolution of $2.9 \AA$ using X-ray crystallography fits left-handed DNA. This biophysical evidence strongly indicated that left-handed DNA could be functionally active.

(e) The hybridization product between an exon and its mRNA cannot wind plectonemically. Many EM pictures clearly indicate that only an exon gene can bind with mRNA in a wide spectrum of biological organisms [30,31]. As shown in Figure 9, the intron is not recognizable by mRNA. 


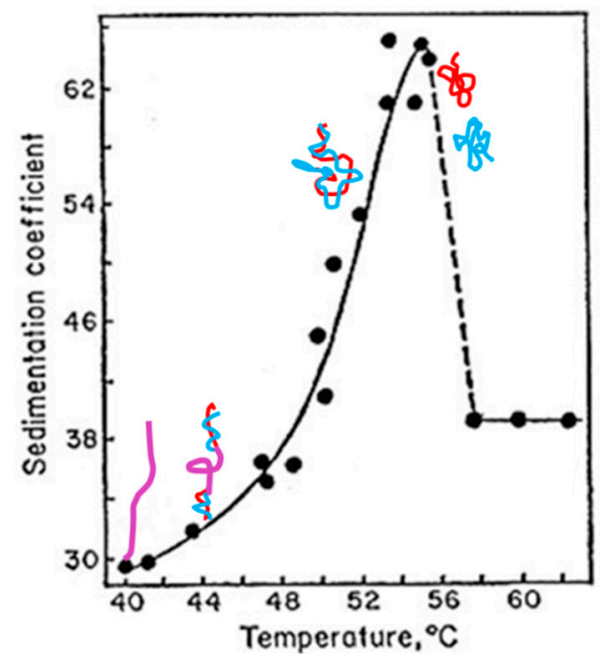

Figure 8. Schematic showing the denaturing process of T7 DNA. The original data are from Freifelder and Davidson [28].

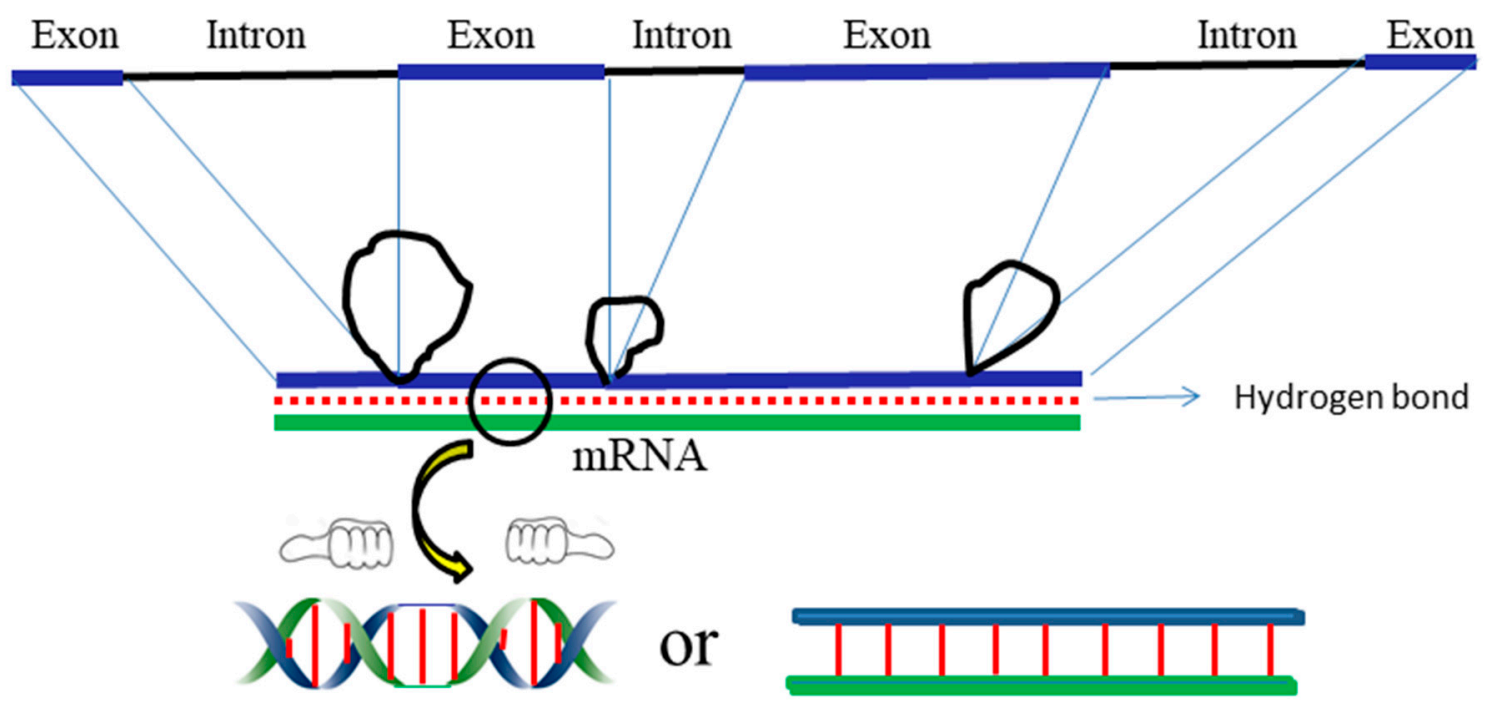

Figure 9. Schematic detailing the hybridization product of exon and mRNA.

\section{Discussion}

By virtue of the rapid advances in molecular biology, more and more information hidden in the genes of various organisms have been deciphered. The completion of the Human Genomic Project showed that the blueprint of a person can be determined. It has greatly impacted the fields of medicine, biotechnology, and life sciences. Important information closely related to public social life have come from the correct reading of the primary structure of DNA. For example, DNA profiling became a key piece of evidence in courts, the production and dispute over genetically modified organisms (GMO), the development of gene therapies, etc.

Dichotomy tells us that the double helix has its side effects or defects. Many students and experts were unintentionally or imperceptibly misguided by the double helix. Hence, the side effect of the double helix on the scientists' psychology is probably indescribable and impalpable. Their implicit bias is hard to cure. Accompanying the great success of DNA sequencing and many amazing advances in molecular biology, overpraise and dazzling glory pushed the double helix model to irrational heights. This caused the public, as well as many scientists and journal editors, to lose the ability to question and doubt the double helix. This deterred Robert Chambers from further investigating his unexpected 
finding and prevented T. T. Wu, among other scientists to get grant support [32,33]. Perhaps, it also hindered many other individual scholars to produce or publish unorthodox ideas or findings.

In contrast to the primary structure of DNA, the attention on the secondary structure of DNA is pitiful. Most people assumed that in nature, the DNA structure is in the B-DNA form that had been convincingly proven using X-ray crystallography. It became a doctrine written in all textbooks. As a rare supplement (see Supplementary Materials), Z-DNA was also proven by X-ray crystallography. This dote sign had better to be changed as an exclamation mark.

Nobody doubts the result obtained from X-ray crystallography! The problem is that all X-ray crystallography evidence was obtained from short fragments of DNA. It is plausible that extrapolating the correct result from short fragments to long DNA is incorrect. Just as someone focusing on a leaf may not see the tree and even less the forest. As a microscopic method, X-ray crystallography is not suitable to determine the molecular structure of very long DNA.

Our investigation uses the mathematical rule, linking number Lk is always equivalent to the sum of total twist of the double helix $\mathrm{Tw}$ and the writhing number $\mathrm{Wr}$, i.e., $\mathrm{Lk}=\mathrm{Tw}+\mathrm{Wr}$, to analyze and evaluate the experimental phenomenon observed from covalently closed circular DNA. It is a macroscopic method. The conclusion derived from experiment reflects the property of the whole plasmid molecule. As shown above, all specially designed experimental results and many facts obtained from various laboratories are consistent with each other. The evidence is self-consistent. This constitutes a chain of evidence sufficient to deny one of the main claims of the double helix, i.e., the two DNA strands are always winding right-handedly or plectonemically.

Similar to a court debate, all true evidence has to be put on the table for careful analysis. While judging the suspected secondary structure of DNA, all evidence collected from both microscopic and macroscopic methods should be fairly considered with no bias.

Different from the idea of God or ghosts, the structure of DNA is supposed to be an objective reality and a recognizable entity. No matter from when, where, or whom a correct DNA structure originates, it should be supported by all evidence that is reasonable and self-consistent.

What really is DNA? In the eyes of human beings, pure DNA is just some kind of white stuff or transparent fiber. Whereas, in the minds of different generations of people, guided by the most knowledgeable scholars at that time, DNA differs greatly. The Watson-Crick double helix model proposed on insufficient evidence in 1953 was reasonable and admirable. The history of DNA repeatedly tells us that our knowledge has to be changed according to the available evidence. The advancement of molecular biology is so fast that it pushes us to update incorrect scientific knowledge or change inappropriate conceptions.

Scientific knowledge is non-rivalrous and non-excludable, whereas different claims or hypotheses may be rivalrous and exclusive. All human knowledge is partial; nobody can reach the final truth. There is probably no standard answer to the question of "what is DNA". Based on available evidence, a provisional answer closer to the truth is appropriate for the moment. We can see something further than before just because we are standing on the shoulders of many great scientists. Nobody complains that there are defects or incorrect information in the canonical double helix model since knowledge comes from evidence and collecting evidence needs time, chance, and vision.

Presently, all evidence shows that native DNA under physiological conditions is dynamic, polymorphous, and ambidextrous, rather than a monotonous plectonemic right-handed double helix.

The plectonemic DNA model seems to go against Occam's razor, i.e., the simplest solution tends to be the correct one. However, as Crick pointed out, implementing Occam's razor in biology can be very dangerous [5]. Unlike cellulose or tendons, DNA is the critical macromolecule of all living cells responsible for various important biological functions; its structure should be very stable to preserve the fidelity of its genetic code and flexible for the convenience of code recognition. It is probably the reasonable choice of nature after billions years of evolution, although the exact reason is unclear.

In the eyes of future historians, ambidextrous DNA is just a slight modification of the double helix, i.e., from plectonemic winding to ambidextrous winding. The rational core of the double helix is 
unchanged, only the incorrect part about the winding of the two strands was amended. However, it is a big leap forward in the conception of the secondary structure of DNA and consequently has a big impact on DNA topology.

The most easily noted difference between two DNA models is on the understanding of supercoiling.

More than 50 years ago, while an extrachromosomal DNA, the polyoma virus DNA was studied in the laboratories of Dulbecco and Vinograd. They found this DNA is circular and resistant to heat or formamide denaturation. The purified DNA contains three components that were named DNA I (supercoiled DNA), DNA II (nicked DNA), and DNA III (linear DNA) afterwards. Vinograd and his colleagues realized that DNA I, normally found under experimental conditions, is a negatively supercoiled DNA; they also assumed it is due to the number of winding turns between two strands less than that of its relaxed counterparts in B-DNA form [34].

A reasonable deduction is that in positively supercoiled DNA, the two strands should be over-wound. The question is how many water molecules can be squeezed out in a positively supercoiled plasmid under physiological conditions. Although B-DNA can be dehydrated in air and turned into more compact A-DNA, it does not mean the two strands can freely tighten with no limits in aqueous solution because the atoms are incompressible and many water molecules are tightly hydrogen-bonded with DNA [35]. The highly positively supercoiled plasmid found by D. Lockshon and D.R. Morris is unexpected [36]. It triggered a question: can a hyper-positively supercoiled plasmid be made as that of a hyper-negatively supercoiled plasmid [37-39]? Nobody knows how the two strands are over-wound in the positively supercoiled DNA if the DNA is in the double helix model.

However, according to the ambidextrous DNA model, the linking number of a relaxed plasmid is zero. The linking numbers of positively or negatively supercoiled plasmids must be much higher than that of their relaxed counterparts. As shown in Figure 10, schematic drawings of the plasmid in two DNA models are put on a two-dimensional plane to represent the big difference between them.

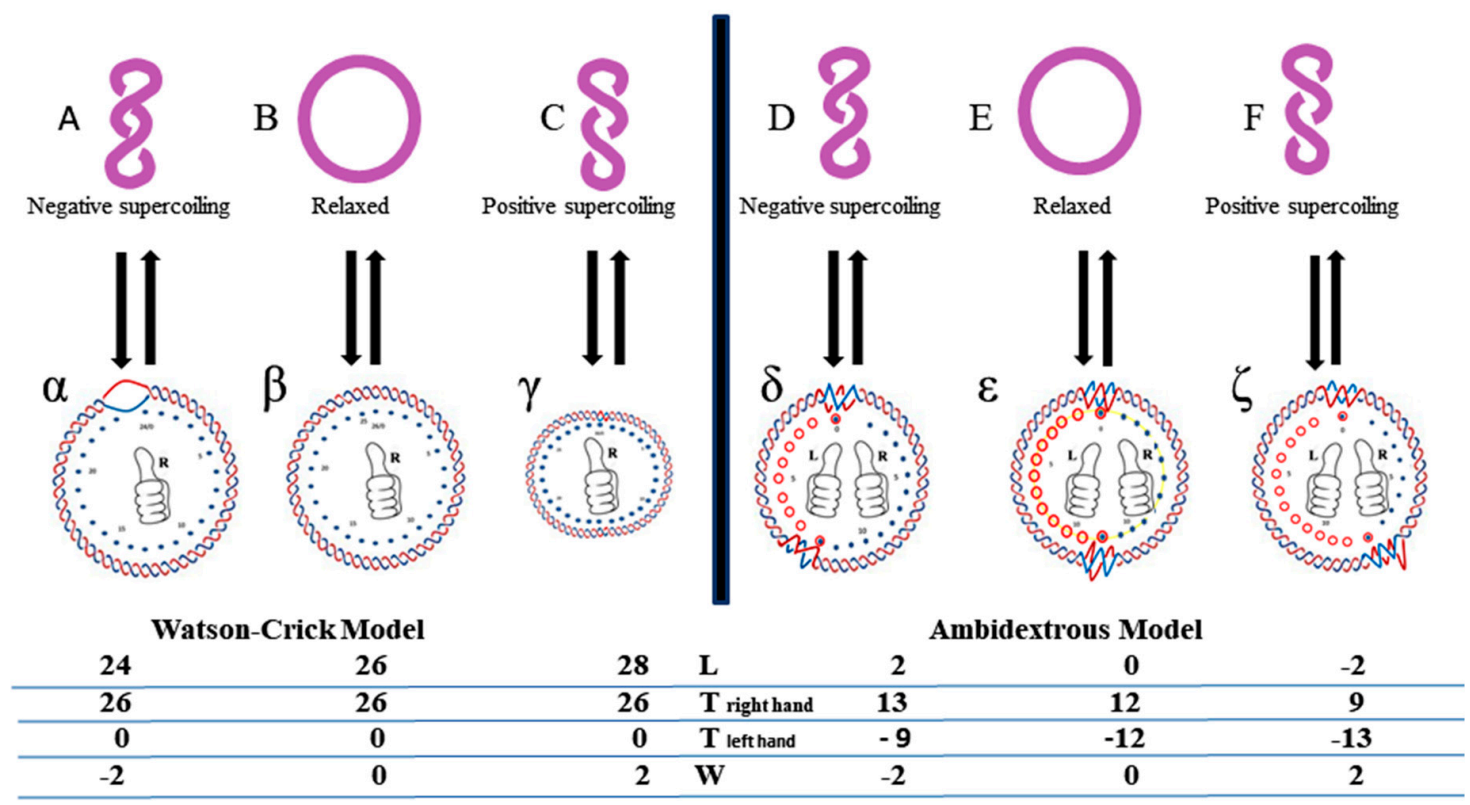

Schematic drawing of two kinds of DNA model

Figure 10. Schematic presentation of two kinds of DNA models.

In this simplified drawing of a 260-bp circular DNA, all forms of DNA are designed with $10 \mathrm{bp}$ per turn. In the pictures, A, B, C, D, E, F are the general profiles of the plasmid; $\alpha, \beta, \gamma, \delta, \varepsilon, \zeta$ are the detailed images of six pairs of fully expanded circular DNAs projected onto a two-dimensional plane. The two complementary circular strands are always hydrogen bonded except there is no hydrogen bond in the loop region in Figure 10A. 
The finding of a zero-linking-number topoisomer indicated that the number of the two opposite-winding turns of that molecule should be the same, i.e., $T_{\text {right-handed }}=\mathrm{T}_{\text {left-handed }}$. The cancellation of the equal amount of opposite winding strands lead to the non-linkage of the two ssc DNAs. i.e., $\mathrm{L}_{\mathrm{k}}=0$. A reasonable deduction is that in a negatively supercoiled plasmid, the turns of the right-handed DNA should be more than that of the left-handed DNA, i.e., $\mathrm{T}_{\text {right-handed }}>\mathrm{T}_{\text {left-handed }}$. Likewise, in a positively supercoiled plasmid, the case is reversed, i.e., $\mathrm{T}_{\text {right-handed }}<\mathrm{T}_{\text {left-handed }}$.

A second suggestion from the finding of a zero-linking-number topoisomer is that the left-handed DNA is a routine DNA, as common as the right-handed DNA. Left-handed DNA is no more limited to Z-DNA, which has a relatively strict sequence requirement of alternative purine and pyrimidine. An independent investigation on pBR322 pointed out that the helical repeats of left-handed DNA is 12 base pairs per turn, which matches the data of Z-DNA obtained from X-ray crystallography [40]. However, the two strands of left-handed DNA are not more tightly tangled with each other, which is not consistent with the expectation of the canonical double helix model. Further investigation may show whether it is coincidental. In short, it is safe to say that Z-DNA is just a member of the left-handed family.

These deductions violated an old concept in DNA topology. It seems a new definition has to be set up to substitute the old one. For example, according to the Watson-Crick model, in a plasmid with $\mathrm{N}$ base pairs, DNA is supposed to have 10 base pairs per turn and the linking number of a relaxed plasmid should be a big integer, i.e., $\mathrm{L}_{0} \approx \mathrm{N} / 10>>0$. The superhelical density $\sigma$ is defined as the linking number difference between supercoiled DNA $(\mathrm{L})$ and relaxed DNA $\left(\mathrm{L}_{0}\right)$ divided by the linking number of relaxed DNA, i.e., $\sigma=\left(\mathrm{L}-\mathrm{L}_{0}\right) / \mathrm{L}_{0}$. However, it would be unreasonable to put zero as a denominator to indicate the superhelical property of the plasmid if the linking number of the relaxed DNA is zero.

A superhelical index, $\mathrm{Si}$, was proposed as a convenient compromise to indicate the superhelical property of a circular DNA [24]. Si is related to only two experimentally measurable parameters: the writhing number $\mathrm{W}_{\mathrm{r}}$ and the base pair number $\mathrm{N}$ of the plasmid, i.e., $\mathrm{Si}=\mathrm{W}_{\mathrm{r}} /(\mathrm{N} / 10.4)$. Si can be used as an index to indicate and compare the supercoiling of different plasmid molecules in any DNA model. The reported $\sigma$ value can still be used as the Si value.

Some readers may ask: if the plasmid DNA is actually following the ambidextrous model, what about linear DNA?

From the experiment of Depew and Wang [41], it is understandable that before and after the ligation, the winding state of the two strands is unchanged. Hence the two strands of linear DNA should always be winding ambidextrously. This statement may cause great confusion or worry. A possible explanation is that the tertiary structure of DNA is dynamic and polymorphous. It can adopt its most probable shape under the provided conditions.

The aim of the proposed ambidextrous DNA model is to try to solve the untangling problem in DNA replication. Evidently, if the two strands of circular E. coli chromosomal DNA are not always tightly tangled with each other, their quick separation would have no topological difficulty. The puzzling problem regarding the quick unwinding of DNA disappears radically.

It is probable that the new DNA model can also help explain the mechanism of CRISPR (clustered regularly interspaced short palindromic repeats). During the long evolution process, bacteria developed a protection against invading viral DNA. CRISPR can be applied for precise and efficient genome editing [42]. The mechanism of it is rather complicated. A critical step is that bacteria's gRNA can easily and accurately find its target on the virus and tightly bind to it for its helper enzyme (Cas9) to snip the virus gene. The problem is how the gRNA, about 20 nucleotides long, can find the correct sequence hidden inside the two turns of viral DNA. Just like searching huge crowds, if everybody is tightly masked, a clever scout drone would be unable to identify or distinguish the terrorist. It would be much easier for the gRNA to search its matching DNA sequences if the two strands of the virus gene are not always tightly tangled with each other, as required in the Watson-Crick model.

As the main genetic message carrier, the secondary structure of DNA found in plasmids should not be limited to prokaryotes. 
In eukaryotes, DNA is further wound on histones. The coding message becomes more difficult to decipher if the DNA is really in the canonical double helix model. While the genetic code is tightly hidden in the chromosome, the question regarding how it can be read by similar homeobox proteins at specific times and places remains un-answered [43]. The ambidextrous DNA model enables easier recognition of the message hidden inside DNA.

The finding of a zero-linking-number topoisomer is a crucial fact that serves as a touchstone for the judgement of two different hypotheses. It acts as a fatal weapon on the Achilles heel of the double helix model, since its occurrence cannot be explained by the canonical double helix model.

The ambidextrous DNA model is not perfect or irrefutable. If the ambidextrous model is correct, a reasonable consequence is that, in any circular DNA, there is at least a pair of transitional sections between the oppositely wound helixes. According to the finding of Ha et al. [44], there are two extruded bases at the junction between the B-DNA and Z-DNA of a 15mer oligonucleotides found using X-ray crystallography. This possibly implies that in any circular DNA, there are at least four pairs of bases that lost their hydrogen bonds at the two junctions.

This may be one of the possible refutations to the ambidextrous model. However, it should be noted that under physiological conditions, DNA structure is dynamic. Its tertiary structure is frequently affected by the surrounding conditions, including temperature, solvent, $\mathrm{pH}$, cations, intercalating reagents, etc. It is unlikely that 8 or 12 deprived hydrogen bonds at the two junctions can be freely moving alone in the circular DNA. Furthermore, it is unfavorable for the stability of a circular DNA to always to keep some unpaired regions. Perhaps the extruded bases found in that short linear DNA can only occur under static conditions during crystallization.

It is probable that our hypothesis and related claims are not easy to be accepted by many readers. We welcome any rational criticism or accusation. It is the normal process of the self-adjustment of science based on testable explanations and predictions. The clash of different ideas or opinions may produce valuable products and is beneficial to the advancement of science.

The strength of a scientific theory is not only in that it can reasonably explain many facts, but also correctly predict what will happen or forecast some previously unknown phenomenon. Crick told us: "The job of theorists, especially in biology, is to suggest new experiments. A good theory makes not only predictions, but surprising predictions that then turn out to be true. (If its predictions appear obvious to experimentalists, why they need a theory?)" [5].

We predicted that there is a zero-linking-number topoisomer in any kind of plasmid [21]. One way of testing the ambidextrous double helix is to examine whether this prediction is correct.

To most scientists, this double helix conjecture is not obvious or even impossible. If proven, it would have surprising effects and would ease the understanding of the ambidextrous DNA model amongst scientists, as well as the public with no background knowledge of DNA topology. Now, we can provide a rather detailed and promising research plan for scientists to prove it or disprove it.

\section{Promising and Feasible Research Proposal}

A large amount of evidence leads to refuting one of the main claims of the double helix model, i.e., the two strands of native DNA are always wound plectonemically. Although the ambidextrous DNA model has got the support of much independent evidence, it would be better if one of its predictions can be verified.

Based on many years of trial and error tests, we learned that the two strands of a plasmid can be efficiently dissociated under very mild conditions [24]. This paves the way for the demonstration of the double helix conjecture.

Actually, this is the test Crick et al. originally proposed in 1979. They assumed that if the two complementary DNAs of a plasmid are unlinked, simply heating can dissociate the two circular DNA strands. However, all native plasmid samples occur as a group with different linking numbers; therefore, it is a prerequisite to find out which one is a potential candidate and then get enough samples to do the test. 
To find a non-linked plasmid or zero-linking-number topoisomer is not easy. The critical point is where can it be found and how can it be found. According to our experience, the zero-linking-number topoisomer is among the relaxed DNA. By following the procedures described here, the double helix conjecture can be verified. A dedicated scientist can accomplish the project with a low investment of time and money in most biochemical laboratories. A detailed description of the operation can be found from the references marked in parenthesis.

1. Choose a plasmid as the research object and make enough pure supercoiled DNA sample.

2. Make a set of relaxed topoisomers from the plasmid. This can be achieved via three different ways:

(a) Relaxing the supercoiled DNA with topoisomerase 1 under different conditions by varying temperature, ethidium bromide (EB)EB concentration, positive ions concentration, etc. [41,45].

(b) Turning the supercoiled plasmid into singly nicked DNA and then ligating them with DNA ligase under different conditions by varying the temperature, EB concentration, positive ions concentration, etc. [41,46].

(c) If a special strain is available, the whole set of topoisomer can be obtained using a novobiocin treatment [36]. This would be the most convenient way to get whole set of relaxed plasmids.

3. The critical step is to completely denature the whole set of relaxed topoisomers slowly and carefully by incubating the plasmid in pure water at an appropriate temperature for a given time [24].

4. The denatured plasmid can be renatured in the appropriate solvent, salt concentration, and temperature for a given time. This step may be the most time consuming. It needs to be tested several times to find the best reproducible condition [24].

5. After the plasmid passes the manipulation of denaturing and renaturing, the two-dimensional AGE with control (same sample before manipulation) can be done. A zero-linking-number topoisomer can be located at the same location of control as that of the only missing band on the agarose gel. A schematic drawing is presented in Figure 11 and the linking number of each band is marked beside.

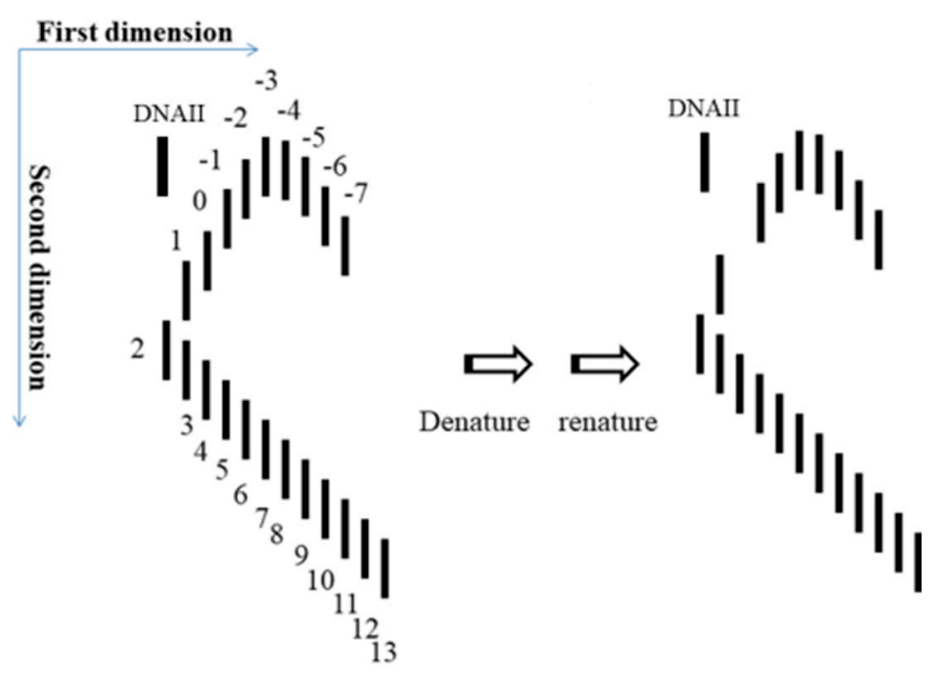

Figure 11. Expected test to find a zero-linking-number topoisomer.

Once the location of the zero-linking-number topoisomer is found, further purification of it may not be necessary. This is because this test already clearly indicated that the missing topoisomer must be a non-linked plasmid. However, if sufficient non-linked plasmids are sample is available, performing the test suggested by Crick et al. becomes feasible Additionally, the pure topoisomer may be of interest to some scholars for further investigation regarding its physical and chemical properties. The data of melting temperature (Tm), circular dichroism (CD) Tm, CD, etc., obtained from it or other individual topoisomers, would help us to get more information from DNA. 
Two useful reminders are provided for those who may try this experiment: (a) During the denaturing process, DNA concentration plays an important role. The unlinked ssc DNA should separate better at a lower DNA concentration, whereas other denatured paired ssc DNAs are not affected by DNA concentration. (b) The binding force of hydrogen bonds between two complementary strands of DNA is closely related to the temperature, whereas the repelling force of the two strands in low salt solution or pure water is not.

Supplementary Materials: The following are available online at http://www.mdpi.com/2073-8994/11/12/1445/s1, From personal communication, Seligmann told me "The unexplained disappearing in gel of DNA occurs frequently, and people dismiss it and repeat the experiment until all samples are where they are supposed to be on the gel. Nobody writes about these disappearances, thinking these are due to artifacts." This is circumstantial evidence in support of my previous experimental finding that plasmids can turn invisible on gel due to dissociation.

Funding: This research received no external funding.

Acknowledgments: The author is grateful to H. Bremer, D. Lang, L. Terada, J. C. Wang, X, Y. Liu, and Q. L. Li for their encouragement and help. Thanks to X. Z. Shen and H. Seligmann for their careful reading of this manuscript and valuable advice.

Conflicts of Interest: The author declares no conflicts of interest.

\section{References}

1. Crick, F.H.C.; Wang, J.C.; Bauer, W.R. Is DNA really a double helix? J. Mol. Biol. 1979, 129, 461-499. [CrossRef]

2. Watson, J.D.; Crick, F.H.C. A structure for deoxybibose nucleic acids. Nature 1953, 171, 737-738. [CrossRef] [PubMed]

3. Rich, A. The excitement of discovery. Ann. Rev. Biochem. 2004, 73, 1-37. [CrossRef] [PubMed]

4. Wang, C. Untangling of the Double Helix; Cold Spring Harbor laboratory Press: New York, NY, USA, 2009.

5. Crick, F.H.C. What Mad Pursuit, A Personal View of Scientific Discovery; New York Basic Books: New York, NY, USA, 1988.

6. Meselson, M.; Stahl, F.W. The replication of DNA in Escherichia coli. Proc. Natl. Acad. Sci. USA 1958, 44, 671-682. [CrossRef]

7. Cairns, J. The bacteria chromosome and its manner of replication as seen by autoradiography. J. Mol. Biol. 1963, 6, 208-213. [CrossRef]

8. Weil, J.H.; Vinograd, J. The Cyclic Helix and Cyclic Coil Forms of Polyoma Viral DNA. Proc. Natl. Acad. Sci. USA 1963, 50, 730-738. [CrossRef]

9. Dulbecco, R.; Vogt, M. Evidence for a ring structure of polyoma virus DNA. Proc. Natl. Acad. Sci. USA 1963, 50, 236-243. [CrossRef]

10. Blattner, F.R.; Plunkett, G., III; Bloch, C.A.; Perna, N.T.; Burland, V.; Riley, M.; Collado-Vides, J.; Glasner, J.D.; Rode, C.K.; Mayhew, G.F.; et al. The complete genome sequence of Escherichia coli K-12. Science 1997, 277, 1453-1562. [CrossRef]

11. Kelman, Z.; O'Donnell, M. DNA polymerase III holoenzyme: Structure and function of a chromosomal replication machine. Ann. Rev. Biochem. 1995, 64, 171-200. [CrossRef]

12. Cyriax, B.; Gath, R.; Cyriax, B.; Gath, R. The Conformation of Double-Stranded DNA. In Naturwissenschaften; Springer Science+Business Media: Berlin, Germany, 1978; Volume 65, pp. 106-108.

13. Rodley, G.A.; Scobie, R.S.; Batest, R.H.T.; Lewitt, R.M. A possible conformation for double stranded polynucleotides. Proc. Natl. Acad. Sci. USA 1976, 73, 2959-2963. [CrossRef]

14. Sasisekharan, V.; Pattabiraman, N.; Goutam, G. Some implications of an alternative structure for DNA. Proc. Natl. Acad. Sci. USA 1978, 75, 4092-4096. [CrossRef] [PubMed]

15. Ullsperger, C.; Cozzarelli, N.R. Contrasting enzymatic activities of topoisomerases IV and gyrase from Escherichia coli. J. Biol. Chem. 1996, 271, 31549-31555. [CrossRef] [PubMed]

16. Berger, J.M.; Gamblin, S.J.; Harrison, S.C.; Wang, J.C. Structure and mechanism of DNA topoisomerase II. Nature 1996, 379, 225-232. [CrossRef] [PubMed]

17. Alberts, B.; Watson, J.; Lewis, J.; Bray, D.; Raff, M.; Roberts, K. Molecular Biology of the Cell, 6th ed.; Garland Science: New York, NY, USA, 2002.

18. Brack, C.; Bickle, T.A.; Yuan, R. The relation of single-stranded regions in bacteriophage PM2 supercoiled DNA to the early melting sequences. J. Mol. Biol. 1975, 96, 693-702. [CrossRef] 
19. Xu, Y.C.; Qian, L.; Tao, Z.J. A hypothesis of DNA structure.-Inspiration from the transformation of supercoiled DNA. Sci. Sin. 1982, 25, 827-836.

20. Xu, Y.C.; Qian, L. Determination of the linking number of pBR322 DNA. Sci. Sin. 1983, 26, 602-613.

21. Xu, Y.C. Replication Demands an Amendment of the Double Helix. In DNA Replication-Current Advances; Seligmann, H., Ed.; InTech: Rijeka, Croatia, 2011; pp. 29-56.

22. Xu, Y.C. Finding of a zero linking number topoisomer. Bachelor Bus. Adm. 2009, 1790, 126-133. [CrossRef]

23. Stettler, U.H.; Weber, H.; Koller, T.; Weissmann, C. Preparation and characterization of form V DNA, the duplex DNA resulting from association of complementary, circular single-stranded DNA. J. Mol. Biol. 1979, 131, 21-40. [CrossRef]

24. Xu, Y.C.; Liu, X.Y. Recent progress in double helix conjecture. Int. J. Appl. Nat. Sci. 2018, 7, 85-100.

25. Dalius, H.; Mantell, N.J.; Alberts, B. Characterization by electron microscopy of the complex formed between T4 bacteriophage gene 32-protein and DNA. J. Mol. Biol. 1972, 67, 341-350. [CrossRef]

26. Bensimon, D.; Simon, A.J.; Croquette, V.V.; Bensimon, A. Stretching DNA with a receiding meniscus: Experiments and results. Phys. Rev. Lett. 1995, 74, 4754-4757. [CrossRef]

27. Lebrun, A.; Lavery, R. Modelling extreme stretching of DNA. Nucleic Acids Res. 1996, 24, $2260-2267$. [CrossRef] [PubMed]

28. Freifelder, D.; Davidson, P.F. Physicochemical studies on the reaction between formaldehyde and DNA. Biophys. J. 1963, 3, 49-63. [CrossRef]

29. McKay, D.B.; Steitz, T.A. Structure of catabolite gene activator protein at 2.9A resolution suggests binding to left-handed B-DNA. Nature 1981, 290, 744-749. [CrossRef] [PubMed]

30. Gilbert, W. Why genes in pieces. Nature 1978, 271, 501. [CrossRef] [PubMed]

31. Chambon, P. Split genes. Sci. Am. 1981, 244, 60-71. [CrossRef]

32. Biegeleisen, K. Topologically non-linked circular duplex DNA. Bull. Math. Biol. 2002, 64, 589-609. [CrossRef]

33. Xu, Y.C. A hypothesis on the secondary structure of DNA. Int. J. Nov. Res. Life Sci. 2015, 2, 50-58.

34. Vinograd, J.; Lebowitz, J. Physical and Topological Properties of Circular DNA. J. Gen. Physiol. 1966, 49, 103-125. [CrossRef]

35. Bastos, M.; Castro, V.; Mrevlishvili, G.; Teixeira, J. Hydration of ds-DNA and ss-DNA by neutron quasielastic scattering. Biophys. J. 2004, 86, 3822-3827. [CrossRef]

36. Lockshon, D.; Morris, D.R. Positively supercoiled plasmid DNA is produced by treatment of Escherichia coli with DNA gyrase inhibitors. Nucleic Acids Res. 1983, 11, 2999-3017. [CrossRef] [PubMed]

37. Pruss, G.J.; Drlica, K. Topoisomerase I mutants: The gene on pBR322 that encodes resistance to tetracycline affects plasmid DNA supercoiling. Proc. Natl. Acad. Sci. USA 1986, 83, 8952-8956. [CrossRef] [PubMed]

38. Pruss, G.J. DNA topoisomerase I mutants. Increased heterogeneity in linking number and other replicon-dependent changes in DNA supercoiling. J. Mol. Biol. 1985, 185, 51-63. [CrossRef]

39. Albert, A.C.; Spirito, F.; Figueroa-Bossi, N.; Bossi, L.; Rahmouni, A.R. Hyper-negative template DNA supercoiling during transcription of the tetracycline-resistance gene in topA mutants is largely constrained in vivo. Nucleic Acids Res. 1996, 24, 3093-3099. [CrossRef]

40. Xu, Y.C. Helical repeats of left-handed DNA. Open J. Mol. Integr. Physiol. 2014, 4, 23-26. [CrossRef]

41. Depew, R.E.; Wang, J.C. Conformational fluctuations of DNA helix. Proc. Natl. Acad. Sci. USA 1975, 72, 4275-4279. [CrossRef]

42. Lander, E.S. The Heroes of CRISPR. Cell 2016, 164, 18-28. [CrossRef]

43. Bürglin, T.R.; Affoller, M. Homeodomain proteins: An update. Chromosoma 2016, 125, 497-521. [CrossRef]

44. Ha, S.C.; Lowenhaupt, K.; Rich, A.; Kim, Y.G.; Kim, K.K. Crystal structure of a junction between B-DNA and Z-DNA reveals two extruded bases. Nature 2005, 437, 1183-1186. [CrossRef]

45. Bauer, W.; Vinograd, J. The interaction of closed circular DNA with intercalative dyes. I. The superhelix density of SV40 DNA in the presence and absence of dye. J. Mol. Biol. 1968, 33, 141-171. [CrossRef]

46. Xu, Y.C.; Bermer, H. Winding of the DNA helix by divalent metal ions. Nucleic Acids Res. 1997, 25, 4067-4071. [CrossRef] [PubMed]

(C) 2019 by the author. Licensee MDPI, Basel, Switzerland. This article is an open access article distributed under the terms and conditions of the Creative Commons Attribution (CC BY) license (http://creativecommons.org/licenses/by/4.0/). 